TENDENCIAS

Revista de la Facultad de Ciencias

Económicas y Administrativas.

Universidad de Nariño

ISSN-E 2539-0554

Vol. XXII No. 1 - 1er Semestre 2021,

Enero-Junio - Páginas 226-253

\title{
LA COMPETITIVIDAD TURÍSTICA. UNA APROXIMACIÓN DESDE EL DEPARTAMENTO DE BOYACÁ, COLOMBIA
}

\section{THE TOURIST COMPETITIVENESS. AN APPROACH FROM THE DEPARTMENT OF BOYACA, COLOMBIA}

\section{A COMPETITIVIDADE TURÍSTICA. UMA ABORDAGEM DO DEPARTAMENTO DE BOYACA, COLÔMBIA}

Ana Milena Serrano-Amado; Luz A. Montoya-Restrepo; Nidia Paola Amado-Cely

Magíster en Administración, Universidad Nacional de Colombia. Docente, Escuela de Administración de Empresas Agropecuarias, Universidad Pedagógica y Tecnológica de Colombia. ORCiD: 00000003-0579-5722. E-mail: ana.serrano@uptc.edu.co, Colombia.

Doctora en Ciencias Económicas, Universidad Nacional de Colombia. Profesora Titular Universidad Nacional de Colombia sede Medellín. ORCiD: 0000-0002-4896-1615. E-mail: lamontoyar@unal.edu.co, Colombia.

Magíster en Economía, Universidad Pedagógica y Tecnológica de Colombia. Docente Escuela de Administración Industrial, Universidad Pedagógica y Tecnológica de Colombia. ORCiD: 0000-00031454-3017. E-mail: nidia.amado@uptc.edu.co, Colombia.

Recibido: 21 de julio de 2020

Aprobado: 23 de septiembre de 2020

DOI: https://doi.org/10.22267/rtend.212201.162 


\title{
Resumen
}

El turismo es un sector económico fuerte en la economía mundial que genera beneficios económicos importantes para los países. Este artículo es resultado de la investigación desarrollada por el grupo CERES. El objetivo de la investigación se centró en la revisión de diferentes modelos de competitividad turística para implementarlos en la Provincia de Sugamuxi, centro de la investigación. Para llegar al cumplimiento de los objetivos, se planteó una metodología cualitativa con un método de análisis documental para destacar los estudios y modelos de competitividad más relevantes para la implementación; lo que generó la aplicación del modelo de Crouch y Ritchie (1999); se identificaron los factores más impactantes para la región. Dentro de los resultados se muestran las fortalezas como el tipo de turismo natural que se genera y dentro de las debilidades la falta de asociación por parte de los actores que intervienen en el sector turístico, encontrando como grandes retos la infraestructura, la información, organización y lazos del mercado, estas debilidades se tienen que mejorar puesto que el sector turístico se está proyectando como clase mundial.

Palabras clave: competencia; desempeño; estrategia; productividad; turismo.

JEL: D4; L1; L25; L14; M1

\begin{abstract}
Tourism is a strong economic sector in the world economy that generates significant economic benefits for countries. This article is the result of research carried out by “Group CERES”. The objective of the research focused on reviewing different models of tourist competitiveness and implementing them in the Province of Sugamuxi, the focus of the research. To reach the objectives, a qualitative methodology was proposed with a method of documentary analysis to highlight the most relevant studies and competitiveness models for implementation; what generated the application of the Crouch and Ritchie (1999) model; the most impactful factors for the region were identified. The results show the strengths such as the type of natural tourism that is generated and within the weaknesses the lack of association on the part of the actors involved in the tourism sector, finding infrastructure, information, organization and market ties, these weaknesses have to be improved as the tourism sector is projecting itself as world class.
\end{abstract}


Keywords: competition; performance; strategy; productivity; tourism.

JEL: D4; L1; L25; L14; M1

\section{Resumo}

O turismo é um setor econômico forte na economia mundial que gera benefícios econômicos significativos para os países. Este artigo é resultado de uma pesquisa realizada pelo grupo CERES. O objetivo da pesquisa centrou-se em rever diferentes modelos de competitividad de turística e implementá-los na Província de Sugamuxi, foco da pesquisa. Para atingir o cumprimento dos objetivos, foi proposta uma metodologia qualitativa com um método de análise documental para evidenciar os estudos e modelos de competitividade mais relevantes para implementação; o que gerou a aplicação do modelo de Crouch e Ritchie (1999); os fatores mais impactantes para a região foram identificados. Os resultados mostram os pontos fortes como o tipo de turismo natural que é gerado e dentro dos pontos fracos a falta de associação por parte dos atores envolvidos no setor do turismo, encontrando infraestruturas, informação, organização e laços de mercado, essas fragilidades precisam ser melhoradas, pois o setor de turismo se projeta como de classe mundial.

Palavras-chave: competição; desempenho; estratégia; produtividade; turismo.

JEL: D4; L1; L25; L14; M1

\section{Introducción}

La competitividad es definida como la disposición que tiene una empresa para la producción y comercialización de los bienes o productos teniendo en cuenta el control de precios, calidad para ser mejores que los rivales. (Porter, 2015, p. 160). Este paradigma ha evolucionado significativamente durante varias épocas, resaltando en cada paso aportes para los mercados y la sociedad en general (Pulido y Sánchez, 2010). Actualmente, es un tema primordial para los países, ya que juega un papel indispensable para medir el crecimiento y desarrollo económico. Considerando la competitividad en el contexto de los destinos turísticos, es definida como "función del atractivo del destino, su gestión, organización, información y eficiencia, señalando, además, que el atractivo depende de los atractores y disuasores presentes; la gestión depende de los esfuerzos de mercadeo y de gerencia" (Castellanos et al., 2014, p. 255). 
Los destinos turísticos tienen grandes posibilidades en el desarrollo de la economía. En el año 2017 este sector económico reportó un crecimiento del 6\%, siendo Oriente Medio (9\%), Europa (8\%), África (8\%), Asia (6\%) y América (3\%) los sitios que presentan mayor receptividad turística (Organización Mundial de Turismo [OMT], 2018). Según los resultados presentados por la OMT (2018) los países con mayor aceptación en el continente americano son Brasil (6,3\%), Argentina $(5,7 \%)$, Chile $(4,4 \%)$, Colombia $(2,98 \%)$ y Ecuador (1,54\%), respectivamente.

En el departamento de Boyacá, donde se desarrolló la investigación, el aporte del PIB del sector turismo fue del 3,2\% para el año 2018, siendo los subsectores como los hoteles y restaurantes los más representativos, generando un total de 1.821 empleos directos (Ministerio de Comercio, Industria y Turismo [MinCIT], 2014, p. 10). La Provincia de Sugamuxi foco de la presente investigación, está conformada por 13 municipios, cuenta con "71 atractivos turísticos, el 77\% corresponde al patrimonio cultural material, el $8 \%$ a patrimonio cultural inmaterial y el $9 \%$ a festividades", donde la tasa de ocupación para el año 2017 fue del 38,2\%, destacándose el turismo natural, religioso, y festividades principalmente.

Existen muchos motivadores para visitar un lugar: descanso, cultura, academia, incluso actividades laborales. La movilidad de las personas activa diferentes sectores de la economía y todos sus servicios asociados, lo que permite un uso de recursos locales para beneficiar el mercado laboral y aumentar los ingresos en la región. Mejorar la competitividad es un gran compromiso para las regiones ya que se garantiza mayor calidad de vida para poblaciones, incluyendo las más vulnerables.

El presente artículo se plantea con el objetivo de realizar un análisis de los diferentes modelos de competitividad turística e implantarlo en la Provincia de Sugamuxi. Para tal efecto, se incorpora una metodología cualitativa desarrollada mediante el método de análisis documental de los diferentes modelos y estudios enfocados en el sector turístico. Además, se implementa el modelo de Crouch y Ritchie (1999) para la Provincia. Como aportación, se resalta las fortalezas y debilidades que se evidencian en esta región en aras de proponer estrategias para mejorar los resultados encontrados en la investigación. 


\section{Referentes teóricos}

Competitividad. Los economistas emplean la competitividad desde la perspectiva macroeconómica. En este marco, la mencionan como un "problema" cuando un país con capacidad de "empleo constante, presentan perdidas de "cuenta corriente", las cuales necesitan intervención de la "deflación y depreciación". Sin embargo, se encuentran definiciones concretas respecto a este término, es vista como una estrategia para centrar los elementos que evitan el crecimiento "económico" en un plazo medio como son la "productividad, innovación, y las habilidades gerenciales" (Lall, 2001, p. 1503).

La política de competitividad en Colombia se encuentra versada en el documento del Consejo Nacional de Política Económica y Social (CONPES 3527) de 2008. En este, se presentan 15 planes de acción para mejorar la competitividad a nivel nacional; estos se dividen en: "sectores de clase mundial, salto de productividad y empleo, competitividad en el sector agropecuario, formalización empresarial, formalización laboral, ciencia tecnología e innovación, educación por competencias laborales, infraestructura de minas y energía, infraestructura de trasporte, profundización financiera, simplificación tributaria, las tecnologías de la información TIC cumplimiento de contratos, sostenibilidad ambiental como factor de competitividad, fortalecimiento institucionalidad de la competitividad" (CONPES, 2008, p. 2). Esta política tiene como objetivo "la trasformación productiva del país "mediante el aumento de la productividad, calidad y trasformación productiva" (CONPES, 2008, p. 6). Los pilares aprobados para el desarrollo de esta política son "desarrollo de sectores o clúster de clase mundial, promoción de la productividad y el empleo, formalización laboral y empresarial, promoción de la ciencia, la tecnología y la innovación, y estrategias transversales (CONPES, 2008, p. 8).

Para el año 2019 los índices de competitividad, publicados por el Foro Económico Mundial, Colombia obtuvo un crecimiento de 4,01 puntos, con lo que logró colocarse en el puesto No. 61 del ranking de competitividad mundial, esto presentó una leve mejoría referente al año 2015. Lo anterior, se puede ver en la Tabla 1 que muestra la evolución de índices de competitividad en Colombia. 


\section{Tabla 1}

Evolución de índices de competitividad en Colombia

\begin{tabular}{lll}
\hline Fecha & Ranking de Competitividad & Índice de Competitividad \\
\hline 2019 & $57^{\circ}$ & 62,73 \\
2018 & $60^{\circ}$ & 61,63 \\
2017 & $66^{\circ}$ & 61,29 \\
2016 & $61^{\circ}$ & 61,12 \\
2015 & $66^{\circ}$ & 60,46 \\
2014 & $69^{\circ}$ & 59,90 \\
2013 & $69^{\circ}$ & 59,76 \\
2012 & $68^{\circ}$ & 60,04 \\
2011 & $68^{\circ}$ & 59,18 \\
2010 & $69^{\circ}$ & 57,91 \\
2009 & $74^{\circ}$ & 57,84 \\
2008 & $69^{\circ}$ & 57,76 \\
2007 & $63^{\circ}$ & 58,57 \\
\hline
\end{tabular}

Fuente: Consejo Privado de Competitividad, 2019.

Teniendo en cuenta que la consolidación del Índice Global de Competitividad (IGC) del Foro Económico Mundial (FEM), se realiza para medir la capacidad que tiene un país de generar oportunidades de desarrollo económico a los ciudadanos, valorando los factores que impulsan la productividad y proporcionan las condiciones para el progreso social y la agenda de desarrollo sostenible, el lograr un adecuado indicador proporciona un termómetro de las condiciones del país para mejorar el desempeño. Es importante aclarar que, a partir de 2018, se realizó un cambio de metodología, por lo que el índice desde esta fecha se enfoca en 12 pilares, divididos en entorno habilitante y mercados (Tabla 2). 


\section{Tabla 2}

\section{Pilares de la Competitividad}

\section{2 pilares de la Competitividad}
Entorno habilitante
1. Instituciones
7. Mercado de bienes
2. Infraestructura
8. Mercado laboral
3. Adopción de TICs
9. Mercado financiero
4. Estabilidad macroeconómica
10. Tamaño del mercado
Capital humano
Ecosistema de innovación
5. Salud
11. Dinamismo empresarial
6. Habilidades
12. Capacidad de innovación

Fuente: Schwab, 2018.

Para 2019, el indicador midió 103 variables específicas. De estas, 47 corresponden a la Encuesta de Percepción realizada a empresarios y 56 son datos duros y estadísticas tomadas de fuentes oficiales de cada economía, de la misma manera el índice se construyó con 103 indicadores: (i) 56 son datos duros y estadísticas tomadas de fuentes oficiales de cada economía, los cuales tienen un peso del $70 \%$ sobre el total de la medición, (ii) 47 corresponden a la Encuesta de Percepción realizada a empresarios, que tiene un peso del $30 \%$ en el índice.

Con esta perspectiva y el interés por mejorar la competitividad en Colombia, se menciona el posicionamiento de la productividad relativa del país, especialmente en los servicios financieros, la agricultura y servicios inmobiliarios. Es por esta razón la importancia de resaltar los principales elementos: poca diversificación y poco valor agregado en los métodos productivos, baja fabricación y capacidad de generar empleo en los sectores formales, igualmente se encuentra la disminución en el sector agropecuario, generando con esto la informalidad empresarial y laboral, en cuanto la innovación y la tecnología se evidencian bajos niveles, en cuanto al mercado se percibe la poca profundidad y sofisticación, faltas en la construcción de transporte y energía, entre otros aspectos (CONPES, 2008, p. 5).

Competitividad Turística. Crouch y Ritchie (1999, p. 23) referencian la competitividad en el sector turístico como "la capacidad de un país para crear valor añadido e incrementar, de esta forma, el 
bienestar nacional mediante la gestión de ventajas y procesos, atractivos, agresividad y proximidad, integrando las relaciones entre los mismos en un modelo económico y social”. Igualmente definen un destino turístico como "el espacio o ámbito geográfico con rasgos propios de clima, cultura, atractivos, infraestructuras" (Crouch y Ritchie, 1999, p. 23). Definen la competitividad de los destinos turísticos como "la capacidad para incrementar el gasto turístico, para atraer visitantes de forma creciente, a la vez que se les proporciona experiencias satisfactorias y se realiza de una forma beneficiosa, mientras que se garantiza el bienestar de la población residente y se preserva el capital natural del destino para las generaciones futuras" (Crouch y Ritchie, 1999, p. 23).

En la Figura 1, se evidencia los resultados acerca de la trayectoria sobre el estudio de la competitividad turística, donde sobresalen 468 estudios desde 1985 hasta el año 2020.

\section{Figura 1}

\section{Estudios sobre competitividad turística}

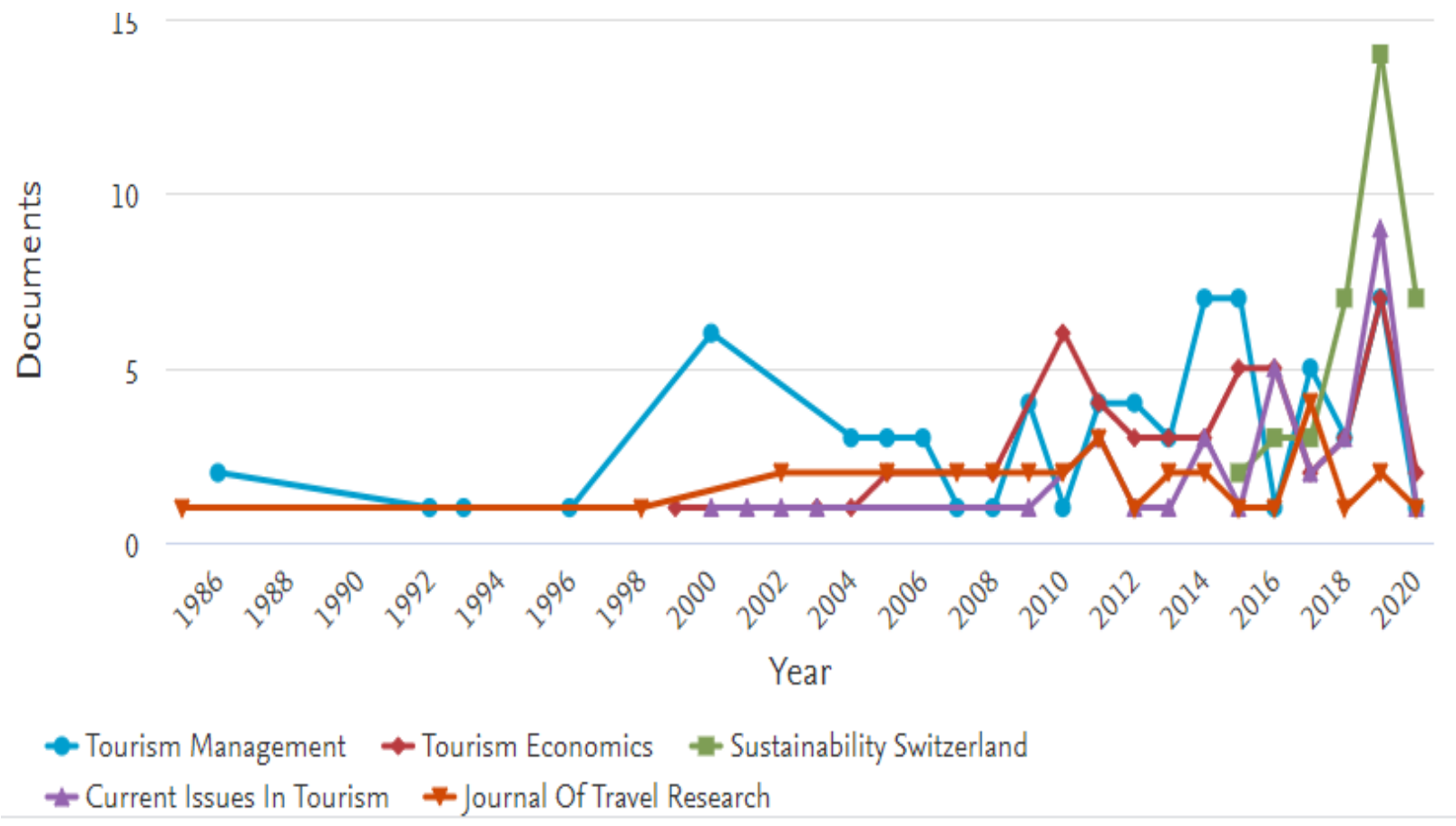

Fuente: Tomado de base de datos Scopus, 2020. 
En la Figura 1 se puede evidenciar la evolución de los estudios relacionados con la competitividad turística, analizado desde tres enfoques, la administración, la economía y la sostenibilidad; resaltando este último como un aporte significativo desde el año 2016.

Igualmente, se puede observar la importancia sobre los estudios de competitividad turística, los que inician en 1985; mientras que toman un auge considerable a partir del año 2006 hasta la fecha actual, como se evidencia con el análisis de los siguientes estudios (Tabla 3):

\section{Tabla 3}

Estudios de competitividad turística

\begin{tabular}{|c|c|c|c|}
\hline AÑO & AUTOR & $\begin{array}{l}\text { NOMBRE DEL } \\
\text { ESTUDIO }\end{array}$ & DESCRIPCIÓN \\
\hline 2006 & $\begin{array}{l}\text { Kozak y Andreu, } \\
2006\end{array}$ & $\begin{array}{l}\text { Progresos en la } \\
\text { comercialización del } \\
\text { turismo. }\end{array}$ & $\begin{array}{l}\text { Cuyo objetivo primordial es evaluar el progreso del } \\
\text { marketing en los destinos turísticos y empresas } \\
\text { individuales, donde se ve reflejado la importancia del } \\
\text { marketing como elemento esencial para la } \\
\text { competitividad del destino turístico. }\end{array}$ \\
\hline 2006 & $\begin{array}{l}\text { Berki y Gonda, } \\
2006\end{array}$ & $\begin{array}{l}\text { Posicionamiento de } \\
\text { escenas urbanas del } \\
\text { turismo cultural en } \\
\text { Hungría. }\end{array}$ & $\begin{array}{l}\text { En este trabajo se realiza una investigación del } \\
\text { posicionamiento del mercado en las ciudades que } \\
\text { poseen calidad en atractivos turísticos, donde se } \\
\text { tienen en cuenta el flujo de huéspedes y se realiza un } \\
\text { análisis de la calidad y capacidad en el servicio de } \\
\text { alojamiento como estrategia crucial para la } \\
\text { evaluación de la ventaja competitiva. }\end{array}$ \\
\hline 2013 & $\begin{array}{l}\text { Bagarić y Žitinić, } \\
2013\end{array}$ & $\begin{array}{l}\text { Competitividad de la } \\
\text { región de Kvarner: } \\
\text { Desafíos para la gestión } \\
\text { de destinos y la marca. }\end{array}$ & $\begin{array}{l}\text { En este estudio el autor refleja la importancia de la } \\
\text { calidad de la prestación de los servicios turísticos } \\
\text { como eje fundamental para el crecimiento de la } \\
\text { competitividad, en este estudio se desarrolla el } \\
\text { modelo integrado de competitividad de destino, } \\
\text { tomando } 85 \text { indicadores como base para realizar una } \\
\text { medición. Como conclusión diagnostican que los } \\
\text { puntos débiles de la competitividad son los índices de } \\
\text { gestión, puesto que los centros turísticos no cumplen } \\
\text { las normas establecidas para el desarrollo, y el punto } \\
\text { fuerte es la diversidad de los recursos turísticos. }\end{array}$ \\
\hline
\end{tabular}

Fuente: elaboración propia, 2019.

Berki y Gonda (2006), Kozak y Andreu (2006) y Bagarić y Žitinić (2013) resaltan la importancia de la eficacia del servicio, los índices de gestión y la diversidad de recursos turísticos como eje 
fundamental para evaluar la competitividad turística. Por otra parte, Chen (2013), Firgo y Fritz (2017) y Andrades et al. (2014) coinciden en que se debe tener claro cuatro pilares para que un destino turístico sea competitivo, dentro de los cuales se tiene la mejora de la prosperidad a largo plazo, maximizar la satisfacción de los clientes y el crecimiento en las empresas locales y mejorar los recursos y los impactos turísticos.

Adicional se plantean los estudios presentados por los autores relacionados en la Tabla 4:

\section{Tabla 4}

Estudios de competitividad turística

\begin{tabular}{|c|c|c|c|}
\hline AÑO & AUTOR & NOMBRE DEL ESTUDIO & DESCRIPCIÓN \\
\hline 2013 & Chen, 2013 & $\begin{array}{l}\text { Factores que influyen en la } \\
\text { calidad del servicio interno en } \\
\text { los hoteles turísticos } \\
\text { internacionales. }\end{array}$ & $\begin{array}{l}\text { En esta investigación se describe el crecimiento que } \\
\text { ha presentado el turismo en los últimos años, } \\
\text { evidencia la exigencia que tienen los turistas a la } \\
\text { hora de servicio de alojamiento, mostrando que la } \\
\text { calidad del servicio es un eje fundamental para el } \\
\text { crecimiento económico de los hoteles, esta } \\
\text { investigación tiene como objetivo realizar un } \\
\text { modelo donde toman como variables la calidad del } \\
\text { servicio interno, la cultura organizacional, } \\
\text { burocracia y el liderazgo, lo que sugieren con este } \\
\text { estudio es crear una cultura organizativa para los } \\
\text { empleados, con el fin de que ellos entiendan que la } \\
\text { autoridad y responsabilidad es indispensable para } \\
\text { generar calidad en la prestación del servicio. }\end{array}$ \\
\hline 2013 & $\begin{array}{l}\text { Firgo y Fritz, } \\
2017\end{array}$ & $\begin{array}{l}\text { ¿Mejora la sostenibilidad la } \\
\text { competitividad de los destinos } \\
\text { turísticos? Evidencia de los } \\
\text { destinos italianos de excelencia. }\end{array}$ & $\begin{array}{l}\text { Para esta investigación aplican el modelo de Richie } \\
\text { y Crouch, donde evalúan la competitividad } \\
\text { mediante indicadores de sostenibilidad, se unificó } \\
\text { los factores de sostenibilidad y el modelo TDC, } \\
\text { donde se analizaron los indicadores más relevantes } \\
\text { para definir la sostenibilidad. Dentro de los } \\
\text { objetivos planteados por los autores está demostrar } \\
\text { que con una "buena política de turismo y gestión de } \\
\text { los destinos más sostenibles, se puede tener un } \\
\text { impacto positivo en la competitividad" (p. 371). }\end{array}$ \\
\hline 2014 & $\begin{array}{l}\text { Andrades et } \\
\text { al., } 2014\end{array}$ & $\begin{array}{l}\text { Tourism } \\
\text { destination competitiveness from } \\
\text { a demand point of view: } n \\
\text { empirical analysis for Andalusia }\end{array}$ & $\begin{array}{l}\text { Este estudio tiene como objetivo realizar un análisis } \\
\text { de la competitividad de los destinos turísticos desde } \\
\text { el punto de vista de la demanda, aplicando el } \\
\text { modelo estructural para los destinos turísticos, } \\
\text { basado específicamente en enfoques teóricos, este } \\
\text { modelo ofrece una explicación sobre los atributos } \\
\text { que ofrecen los destinos turísticos midiendo los } \\
\text { gastos de nivel del destino, como resultado resaltan } \\
\text { que este modelo evalúa la demanda y percepciones } \\
\text { de los turistas frente a los atributos y beneficios que } \\
\text { ofrecen los destinos turísticos. }\end{array}$ \\
\hline
\end{tabular}

Fuente: elaboración propia, 2019. 
Autores como Firgo y Fritz (2017, p. 376) registran que la competitividad del sector turístico debe incluir para generar armonía entre los clientes, bienestar en cuanto a la salud y medio ambiente, resaltando el modelo de Richie y Crouch implementando los indicadores de sostenibilidad enfocándose en el impacto positivo para la competitividad.

En la base de datos Web of Science de los últimos cinco años, se encuentran los siguientes estudios (Figura 2):

\section{Figura 2}

Elementos publicados cada año y cifras
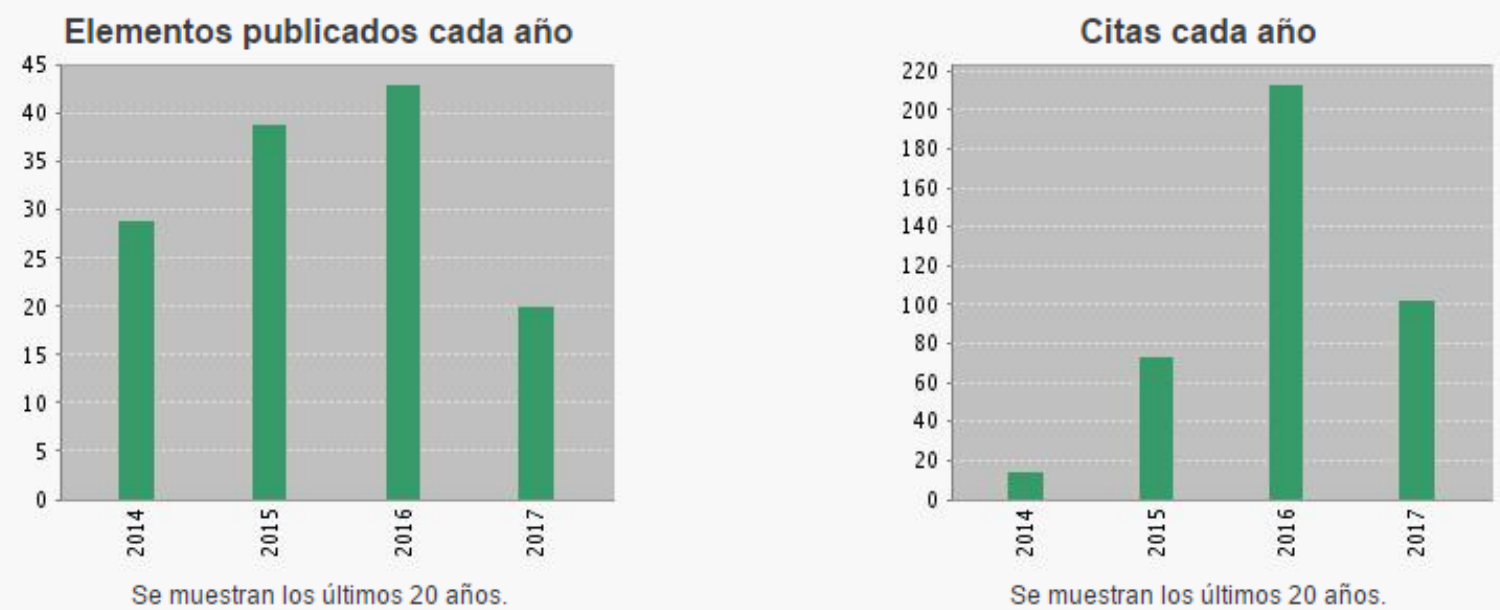

Fuente: Web of Science, 2017.

De esta base de datos, se analizaron 105 estudios, de los cuales se tomaron algunos para realizar los antecedentes de la investigación. Entre ellos, se encuentra el de Alberca y Parte (2013) el cual realizo un análisis de la "eficiencia nacional y regional" (p. 1) de los hoteles españoles, se aplicó una metodología DEA donde se evaluó diversas características y niveles de productividad y competitividad durante los años 2001-2008, como resultados manifiestan que la metodología empleada evalúa los diferentes niveles de productividad, adicional se puede evaluar la "dirección y la gestión empresarial" de las organizaciones especialmente del sector hotelero. 
Otro estudio es el presentado por Ibáñez (2011) quien realizó un análisis enfocado en el diagnóstico del crecimiento competitivo del sector turístico en México, en aras de mejorar el ranking internacional de este país, sus conclusiones destacan las debilidades del sector, en especial, la fragilidad económica, la capacitación del talento humano, la eficacia en los servicios y el apoyo gubernamental en temáticas de información y desarrollo financiero (Ibáñez 2011, p. 24). Por ende, expone que México cuenta con méritos para ser más competitivo en el sector turístico. Menciona que el liderazgo es un eje fundamental para obtener esto, pero deben mejorar en aspectos como "capacitación, promoción, calidad del servicio, certificación laboral, cultura turística, financiamiento y normatividad ambiental” (Ibáñez, 2011, p. 22).

La publicación de Narváez y Fernández (2009) manifiesta unos indicadores de competitividad como "estadísticas de visitantes, contribución a la economía local, prosperidad económica e inversión en turismo". El estudio muestra la falta de compromiso por parte gobierno para optimizar el servicio turístico, ya que cuenta con recursos naturales que pueden ser explotados e incrementar la competitividad del sector.

Urrutia y Cuevas (2016) investigan la composición entre las pequeñas y las medianas compañías para el sector turístico mexicano, así como las conexiones efectivas de varios actores que completan la competitividad. Ellos encuentran que las empresas habrán de trabajar más en colaboración, coordinación e incremento de información" involucrando los diferentes actores como "clientes, estado y empresa privada" (Urrutia y Cuevas, 2016, p. 25). Concluyen que el turismo mexicano presenta baja competitividad por diversos factores como "capacidades de aprendizaje e innovación, la reproducción de iniciativas empresariales exitosas y la conformación de redes” (Urrutia y Cuevas, 2016, p. 30).

Bolaky (2011) realiza un análisis de la competitividad del servicio de alojamiento en el periodo 19952006; aplicado en turistas de Canadá, Estados Unidos y Reino Unido, debido a que son los más frecuentes en visitar el Caribe Americano. Tanto Urrutia y Cuevas (2016) y Bolaky (2011) confirman que la competitividad del sector turístico de América mejoraría si las políticas gubernamentales las favorecieran e implementaran estrategias donde se involucren los diversos actores como son Estado, academia, cliente y organizaciones de orden privado. 
Guzmán et al. (2016, p. 5) realizaron un estudio en los países que acceden a la Alianza del Pacífico (A.P), "cuyo objetivo fundamental es identificar la tendencia en el sector turismo de la economía nacional generadora de innovación en Colombia, México, Perú y Chile, bajo acuerdo macro de vacaciones y trabajo". Para dicho estudio, tomaron como indicadores de competitividad turística "la demanda" para realizar un "análisis multivariable de la innovación del turismo en los países aliados durante los periodos 1999-2013" (Guzmán at al., 2016, p. 8), debido a que el empoderamiento, práctica y conocimiento son ejes fundamentales para que el sector turístico de estos países presente un mayor incremento en cuanto a la competitividad.

En caso particular de Colombia, Polanco (2011) analiza la información entre dos sectores de las redes oriental y suroriente del departamento de Antioquia. Este estudio se realiza con el fin de hacer un análisis de redes sociales indispensable para el desarrollo turístico; comparan las dos subregiones y hacen claridad a la necesidad de gobernanza para que el sector turístico cree más productividad y competitividad. Zuñiga y Castillo (2012) hacen un inventario de las investigaciones y publicaciones realizadas en Colombia en el periodo de 2007-2011; su conclusión es que son pocas las que se han reportado internacionalmente. En este estudio, resaltan que Colombia urge de políticas públicas que desarrollen más la actividad turística como instrumento fundamental para el desarrollo social y económico del país; la necesidad de relacionar actores como "el sector público, el sector privado y la comunidad, además de la participación de las universidades y centros de investigación" con el ánimo del crecimiento del sector turístico en Colombia" (Zuñiga y Castillo, 2012, p. 34); exponen que algunos de los indicadores más importantes para que crezca la competitividad son el recurso humano, mano de obra calificada y afinidad por los viajes.

En el departamento de Boyacá, se encuentra el estudio de Ochoa (2015), quien analiza, la competitividad regional en el municipio de Villa de Leyva como destino turístico a nivel nacional e internacional. Dicha metodología, integra los factores que los componen y mencionan la firma del "Convenio de Competitividad Turística Departamento de Boyacá", en el 2009, que busca una planeación concertada entre el sector público y privado con la siguiente visión: "Para el año 2025 Boyacá estará dentro de los diez principales destinos ecoturísticos y culturales reconocidos a nivel latinoamericano" (Ochoa, 2015, p. 37). En la investigación, hace énfasis a los aspectos que ratifican la baja productividad en el sector turístico resumida en: "Inexistencia de una política pública de 
turismo en el departamento, débil planificación turística en el departamento de Boyacá, débil diseño del producto turístico, deficiencias en la infraestructura básica” (Ochoa, 2015, p. 40).

Se encuentra que la competitividad de los destinos turísticos es un elemento fundamental en el desarrollo de la región y el país. En principio puede ser definida como "función del atractivo del destino, su gestión, organización, información y eficiencia, señalando, además, que el atractivo depende de los atractores y disuasores presentes; la gestión depende de los esfuerzos de mercadeo y de gerencia" (Castellanos et al., 2014, p. 255), en donde se resalta la posibilidad de encontrar elementos atractivos en el cual sea posible atraer visitantes para alcanzar los objetivos propuestos.

Dentro de los modelos se competitividad turística se encuentran los relacionados en la Tabla 5:

\section{Tabla 5}

\section{Modelos competitividad turística}

\begin{tabular}{|c|c|}
\hline Modelo & Descripción \\
\hline Modelo de competitividad de Porter & $\begin{array}{l}\text { Es una herramienta de análisis que permite determinar de qué manera una } \\
\text { empresa puede constituir una ventaja competitiva por medio de cinco } \\
\text { factores }\end{array}$ \\
\hline $\begin{array}{l}\text { Modelo de competitividad del Fondo } \\
\text { Económico Mundial }\end{array}$ & $\begin{array}{l}\text { Identifica la competitividad de un país con la habilidad del mismo para } \\
\text { crear y sostener a largo plazo un valor económico añadido en relación con } \\
\text { sus competidores }\end{array}$ \\
\hline $\begin{array}{l}\text { El modelo de competitividad e } \\
\text { innovación }\end{array}$ & $\begin{array}{l}\text { Menciona que en las últimas décadas los países desarrollados } \\
\text { implementan la innovación para obtener una ventaja competitiva, con el } \\
\text { fin de "incrementar el crecimiento económico sostenible", partiendo de la } \\
\text { creación de nuevos productos, procesos y servicios con el ánimo de } \\
\text { incrementar el valor existente. }\end{array}$ \\
\hline Modelo del World Competitiveness & Para este modelo un ambiente competitivo es aquel que se crea en las \\
\hline Center (WCC) del Institute for & empresas junto con el bienestar, para este modelo es importante la \\
\hline Management and Development (IMD) & $\begin{array}{l}\text { "intervención del Estado" en las decisiones económicas de los países } \\
\text { donde el eje fundamental es el apoyo de la educación y la investigación. }\end{array}$ \\
\hline $\begin{array}{l}\text { Modelo del ISC de la Universidad de } \\
\text { Harvard y el World Economic Forum } \\
\text { - WEF }\end{array}$ & $\begin{array}{l}\text { Presentado por el autor anteriormente mencionado, para este modelo los } \\
\text { pilares de la competitividad están relacionados con "la salud, educación } \\
\text { en todos los eslabones, eficiencia en el desarrollo del trabajo, apoyo } \\
\text { financiero, implementación de tecnología, crecimiento del mercado y la } \\
\text { innovación". }\end{array}$ \\
\hline $\begin{array}{l}\text { Modelo del Sistema Internacional } \\
\text { Integrado de la Competitividad en } \\
\text { Destinos Turísticos (SIIC) }\end{array}$ & $\begin{array}{l}\text { Integrado por cinco factores y sus interrelaciones en el sistema turístico: } \\
\text { los dos primeros, la masa crítica y la naturaleza de la demanda, tienen las } \\
\text { características de crear condiciones básicas para el desarrollo del clúster. } \\
\text { Estos ofrecen una configuración de partida para la planificación } \\
\text { estratégica del mismo }\end{array}$ \\
\hline $\begin{array}{l}\text { Modelo de recursos de Crouch y } \\
\text { Ritchie (1999) }\end{array}$ & $\begin{array}{l}\text { La competitividad dependerá fundamentalmente de los recursos y } \\
\text { atractores básicos y de los factores y recursos de soporte, además de la } \\
\text { existencia de una dirección del destino, elemento clave para mantener una } \\
\text { ventaja competitiva sostenible. }\end{array}$ \\
\hline
\end{tabular}

Fuente: elaboración propia, 2019. 
Para la investigación desarrollada se toma el modelo de Modelo de recursos de Crouch y Ritchie (1999) enfocándose desde el aspecto ambiental para el crecimiento competitivo de los destinos turísticos.

\section{Metodología}

El presente estudio se desarrolla mediante el enfoque cualitativo de análisis documental, el cual se estructuró mediante la ecuación de búsqueda [(competitividad OR «potencial» OR capacidad) AND (turismo OR viajes)] siendo aplicada a bases de datos como Scopus y Web of Science; además, se identifican los estudios más impactantes que se han realizado en los últimos años. A partir de esto, se evidencia la poca investigación en la Provincia de Sugamuxi que analicen la competitividad que genera el sector turístico. Luego se desarrolla mediante el análisis de los diferentes modelos de competitividad turística e incorporación de los factores del modelo conceptual de Crouch y Ritchie (1999, p. 45) adaptado a los trece municipios de la Provincia de Sugamuxi; en ellos, se vincula los factores competitivos realizando una evaluación de los elementos con los respectivos criterios adaptados a cada sector.

Para realizar el análisis de la competitividad de los destinos turísticos en la Provincia de Sugamuxi, fue necesario integrar la metodología del modelo conceptual de Crouch y Ritchie (1999) con los datos e información recolectados, la cual fue consultada y seleccionada en la Secretaría de Turismo de la Gobernación de Boyacá SITUR (2018) y la Cámara de Comercio de Sogamoso (2017), donde se encuentran registrados los municipios de la Provincia de Sugamuxi, analizando aspectos como los recursos principales y complementarios como infraestructura, accesibilidad y recursos facilitadores; posteriormente se toman los factores de administración de recursos basados en marketing, organización, información y servicios, con esta información se aplica el modelo de Crouch y Ritchie (1999), con el cual se identificaron los determinantes de la competitividad en la provincia, ubicación de características en cada elemento fundamental de acuerdo a sus categorías, y finalmente se realizó la evaluación de los determinantes de la competitividad de la Provincia de Sugamuxi como destino turístico. 


\section{Resultados}

Para el análisis de la región, se propone la utilización del modelo de Crouch y Ritchie (1999, p. 276), ampliamente utilizado en investigaciones previas, que referencia la competitividad en el sector turístico como "la capacidad de un país para crear valor añadido e incrementar, de esta forma, el bienestar nacional mediante la gestión de ventajas y procesos, atractivos, agresividad y proximidad, integrando las relaciones entre los mismos en un modelo económico y social", igualmente definen un destino turístico como "el espacio o ámbito geográfico con rasgos propios de clima, cultura, atractivos, infraestructuras, servicios, equipamientos, precio, imagen y gestión, los cuáles, en términos de mercado, se posicionan en la mente de los consumidores” (Ritchie y Crouch, 2003, p. 274).

Con el fin de identificar los factores determinantes de la competitividad de los destinos turísticos en la Provincia de Sugamuxi, se aplica el modelo conceptual en mención (Figura 3) que establece que la competitividad turística está conformada por seis elementos fundamentales:

\section{Figura 3}

Modelo conceptual de Crouch y Ritchie (1999)

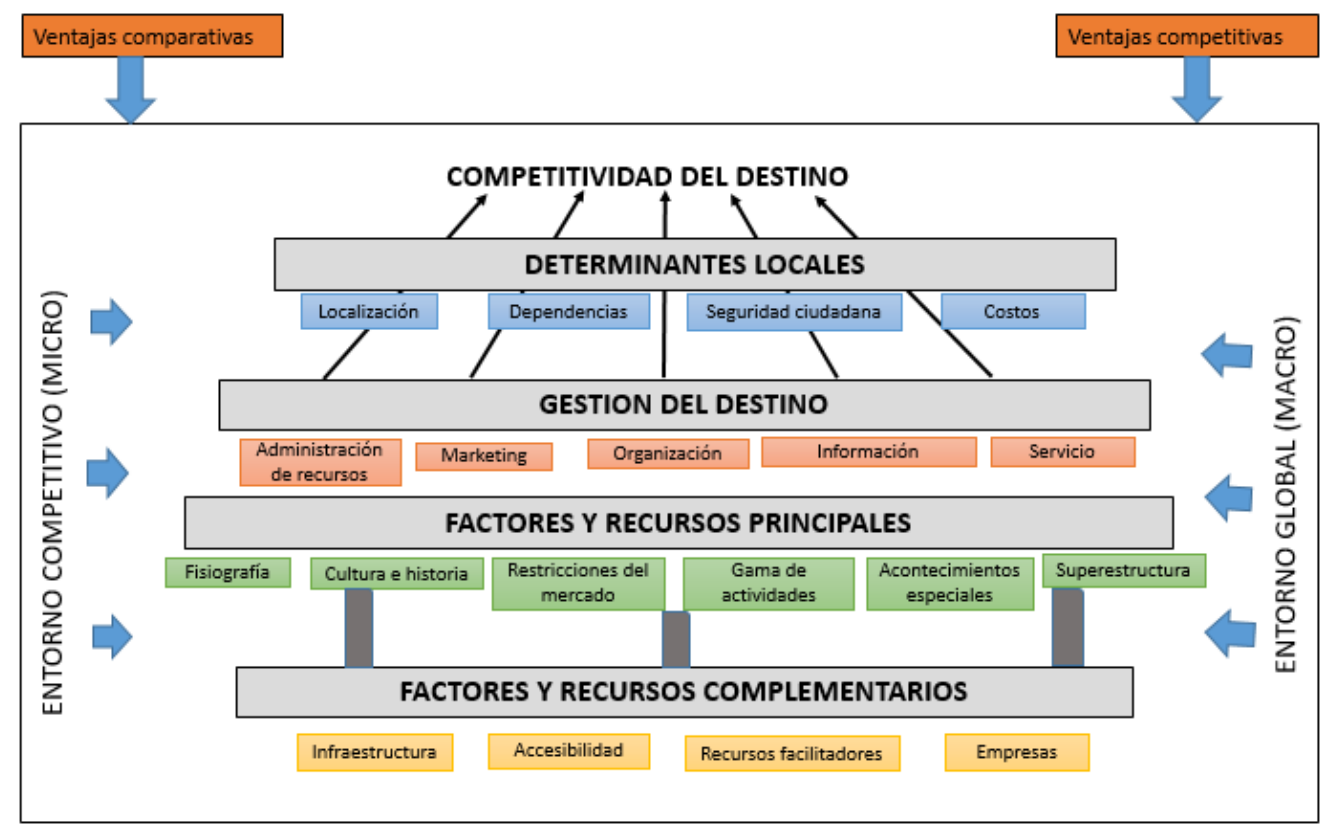

Fuente: Crouch y Ritchie (1999). 
1. El entorno competitivo local (micro), que es el entorno inmediato al que el destino se debe adaptar para competir y está integrado por los diferentes agentes que operan en el sector turístico, como tour-operadores, agentes de viajes, residentes en el destino, empleados, establecimientos hoteleros, instituciones financieras, entre otros.

2. El segundo es el entorno global (macro) que está constituido por aquellas fuerzas externas, por ejemplo, el creciente interés por el medio ambiente, los cambios demográficos en los mercados de origen, la tecnología, políticas gubernamentales.

3. El tercer elemento del modelo de Crouch y Ritchie (1999) como de evidencia en la figura 3 son los "recursos y atractivos principales", que representan los elementos básicos que harán que los visitantes potenciales opten por ese destino concreto y no por otro. En este elemento se agrupan seis grandes categorías: la fisiografía (características paisajísticas, climáticas, etc. del destino), la cultura y la historia (patrimonio monumental e histórico-artístico), las restricciones del mercado, la gama de actividades de ocio, los acontecimientos especiales (Juegos Olímpicos, Exposiciones Universales, Campeonatos Deportivos, etc.) y la superestructura turística (oferta de alojamiento, servicios de restauración, facilidades de transporte, etc.).

4. "Factores y recursos complementarios", entre los que se encuentran las infraestructuras generales del destino (servicios de transporte, servicio de agua, sanidad, etc.), los recursos facilitadores (instituciones financieras, calidad humana de los residentes, recursos de capital y de conocimiento, etc.), la vitalidad y el sentido empresarial y la accesibilidad del destino.

5. El quinto elemento del modelo conceptual es la "gestión del destino", que engloba aquellas actividades que aumentan el interés por los recursos y atractivos principales, que fortalecen la calidad y la efectividad de los factores y recursos complementarios, y que mejor se adaptan a las restricciones impuestas por los determinantes locales del destino. Entre estas actividades pueden citarse las actuaciones de marketing de esfuerzos promocionales, políticas de precios, distribución y comercialización de paquetes turísticos, etc.; la calidad del servicio turístico, los sistemas de información (sobre necesidades de los visitantes, desarrollo efectivo del producto, etc.), estructura 
organizativa (empresarial) del destino y la administración de los recursos (protección y sostenibilidad de los recursos vulnerables a la práctica turística).

6. Finalmente, el último elemento del modelo son los llamados "determinantes locales", los cuales pueden limitar o potenciar, según los casos, la competitividad del destino. Entre estos determinantes se encuentran la propia localización física del destino, las dependencias de otros destinos, la seguridad ciudadana y los costos (el costo de la vida en el destino, los tipos de cambio de las divisas, etc.), tal y como lo ha mencionado en sus estudios.

Para adaptar el modelo de Crouch y Ritchie (1999) y evaluar los factores competitivos de la Provincia de Sugamuxi se hace un análisis de los trece municipios que conforman la Provincia y un análisis de los seis elementos con sus 19 criterios que describe el modelo. Con los resultados obtenidos de esto último, se presentan los siguientes resultados encontrados en las Tablas 6 a 9. 
La competitividad turística. una aproximación desde el departamento de Boyacá, Colombia

Ana Milena Serrano Amado; Luz A. Montoya Restrepo; Nidia Paola Amado Cely

\section{Tabla 6}

Factores y recursos principales de la Provincia de Sugamuxi

\begin{tabular}{|c|c|c|c|c|c|c|c|c|c|c|c|c|c|}
\hline \multicolumn{14}{|c|}{ FACTORES Y RECURSOS PRINCIPALES } \\
\hline FACTOR & & & & & & MUNI & IPIOS & & & & & & \\
\hline Fisiografía & SOGAMOSO & AQUITANIA & CUITIVA & FIRAVITOBA & GAMEZA & IZA & MONGUA & MONGUI & NOBSA & PESCA & TIBASOSA & TOPAGA & TOTA \\
\hline $\begin{array}{l}\text { Lagos, Lagunas, } \\
\text { corrientes de agua }\end{array}$ & & 1 & 1 & & 4 & 1 & 1 & & & & 2 & & 1 \\
\hline Playas, islas & & 2 & 2 & & 1 & & & & & & & & \\
\hline Termales & & & & & & 1 & & & & & & & \\
\hline Páramos, parques & & & & & & & & & & & & & \\
\hline naturales & & 5 & & & 1 & 1 & 2 & 1 & & 1 & 2 & & \\
\hline $\begin{array}{l}\text { Temperatura } \\
\text { promedio }\end{array}$ & $14,2^{\circ}$ & $11^{\circ}$ & $11,5^{\circ}$ & $11,6^{\circ}$ & $14,1^{\circ}$ & $14,2^{\circ}$ & $11,8^{\circ}$ & $11,6^{\circ}$ & $15,6^{\circ}$ & $13,9^{\circ}$ & $14^{\circ}$ & $12,3^{\circ}$ & $11^{\circ}$ \\
\hline $\begin{array}{l}\text { Cultura e } \\
\text { Historia }\end{array}$ & & & & & & & & & & & & & \\
\hline Centros religiosos & 3 & 1 & 2 & 1 & 1 & 2 & 1 & 2 & 4 & 1 & 1 & 1 & 1 \\
\hline Centros históricos & & & & & & & & & & & & & \\
\hline $\begin{array}{l}\text { y coloniales } \\
\text { Centros }\end{array}$ & 8 & 1 & 4 & 4 & 2 & 4 & 2 & 3 & 3 & & 1 & 1 & \\
\hline arqueológicos & 1 & & 1 & & & & 1 & & & & & & \\
\hline $\begin{array}{l}\text { Restricciones del } \\
\text { mercado } \\
\text { Turistas }\end{array}$ & & & & & & & & & & & & & \\
\hline Extranjeros & & & $14,78 \%$ & turistas son ex & jeros que vi & an la $\mathrm{p}$ & incia (Estad & Inidos, Frar & Canadá & lia y Suiz & & & \\
\hline
\end{tabular}

E1 95,21\% corresponden a turistas domésticos, de los cuales el 83,04\% son de departamentos diferentes a Boyacá y el 12,17\% son turistas Boyacenses.

domésticos

Gama de

actividades

Acontecimientos

especiales

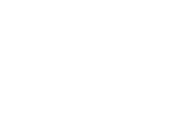

Superestructura

Mincit, Secretaría de Cultura y Turismo de Boyacá, Consejo Departamental Turístico de Boyacá, Consejo de Seguridad Turística de Boyacá, Consejo Regional Turístico, Alcaldías Municipales, ICUTUDER, Prestadores de servicio, Cámara de Comercio de Sogamoso, SENA Sogamoso y Población Anfitriona.

Fuente: creación propia basada en datos de la Secretaría de Turismo de la Gobernación de Boyacá. 


\section{Tabla 7}

Factores y recursos Complementarios de la Provincia de Sugamuxi

\begin{tabular}{|c|c|c|c|c|c|c|c|c|c|c|c|c|c|}
\hline \multicolumn{14}{|c|}{ FACTORES Y RECURSOS COMPLEMENTARIOS } \\
\hline \multirow{2}{*}{$\begin{array}{c}\text { FACTOR } \\
\text { INFRAESTRUCTURA }\end{array}$} & \multicolumn{13}{|c|}{ MUNICIPIOS } \\
\hline & SOGAMOSO & AQUITANIA & CUITIVA & FIRAVITOBA & GAMEZA & IZA & MONGUA & MONGUI & NOBSA & PESCA & TIBASOSA & TOPAGA & TOTA \\
\hline Agencias de viajes & 8 & 5 & 1 & 0 & 1 & 1 & 0 & 3 & 3 & 0 & 1 & 1 & 1 \\
\hline \multicolumn{14}{|l|}{ Bancos y Cajeros } \\
\hline automáticos & 20 & 9 & 1 & 2 & 2 & 2 & 1 & 1 & 3 & 2 & 3 & 1 & 1 \\
\hline Hoteles & 24 & 21 & 5 & 3 & 1 & 10 & 0 & 5 & 6 & 3 & 13 & 4 & 5 \\
\hline Restaurantes & 260 & 43 & 3 & 12 & 3 & 20 & 3 & 13 & 47 & 8 & 54 & 6 & 9 \\
\hline \multicolumn{14}{|l|}{ Bares y centros } \\
\hline nocturnos & 41 & & & & & & & & 5 & & 2 & & \\
\hline \multicolumn{14}{|l|}{ ACCESIBILIDAD } \\
\hline Aeropuerto & \multicolumn{13}{|c|}{ un aeropuerto comercial para aeronaves pequeñas que conecta con departamentos como Casanare, Arauca, parte de Santander y Cundinamarca. } \\
\hline Ferrocaril & \multicolumn{13}{|c|}{ líneas de los ferrocarriles privados que nutren a las factorías } \\
\hline & \multicolumn{13}{|c|}{ cuenta con vías principales, secundarias y terciarias. Todas las carreteras auxiliares de los 13 municipios de la provincia conectan al municipio de Sogamoso y todas confluyen en la } \\
\hline Carreteras & \multicolumn{13}{|c|}{ autopista Tunja-Briceño-Bogotá } \\
\hline Comunicación & \multicolumn{13}{|c|}{ Cuenta con servicio de Internet, telefonía local y telefonía móvil. Además, de un canal comunitario, MoniTV y tres emisoras locales Hit Stereo, Policía Nacional y La Consentida. } \\
\hline Empresas de transporte & 40 & 2 & 1 & 1 & 1 & 1 & 1 & 1 & 2 & 1 & 2 & 1 & 1 \\
\hline \multicolumn{14}{|l|}{ RECURSOS } \\
\hline \multicolumn{14}{|l|}{ FACILITADORES } \\
\hline Operadores turísticos & 11 & 6 & 5 & 3 & & 4 & & 2 & 4 & & 3 & & \\
\hline \multicolumn{14}{|l|}{ Puntos de información } \\
\hline turística & & 2 & & & & 1 & & 1 & 1 & & 1 & & \\
\hline EMPRESAS & 2137 & 41 & 12 & 30 & 33 & 25 & 40 & 40 & 232 & 44 & 109 & 45 & \\
\hline
\end{tabular}

Fuente: creación propia basada en datos de la Secretaría de Turismo de la Gobernación de Boyacá. 
La competitividad turística. una aproximación desde el departamento de Boyacá, Colombia

Ana Milena Serrano Amado; Luz A. Montoya Restrepo; Nidia Paola Amado Cely

\section{Tabla 8}

Gestión del Destino de la Provincia de Sugamuxi

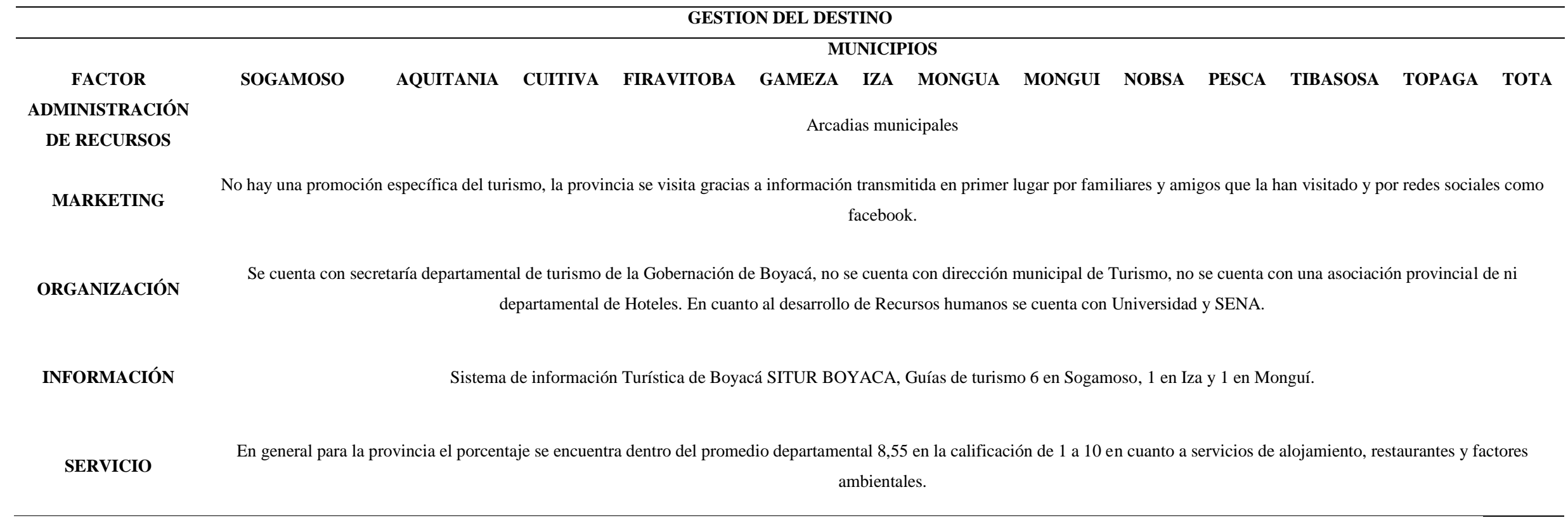

Fuente: creación propia basada en datos de la Secretaría de Turismo de la Gobernación de Boyacá. 


\section{Tabla 9}

Determinantes Locales de la Provincia de Sugamuxi

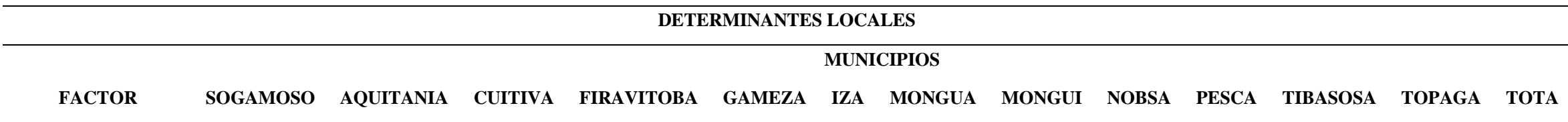

LOCALIZACIÓN Se ubica en el centro del departamento de Boyacá a tres horas de Bogotá, Desde Tunja se toma la carretera central del norte, se pasa por Paipa y Duitama, para llegar al Valle de Sogamoso, entrando por Tibasosa o por Nobsa para llegar a Sogamoso que es la capital de la provincia.

DEPENDENCIAS

SEGURIDAD

CIUDADANA

Costos
Secretaría de Turismo de Boyacá y alcaldías municipales

La percepción de seguridad ciudadana y tranquilidad en la provincia en general es de 8,97 en la valoración de 1 a 10 .

Fuente: creación propia basada en datos de la Secretaría de Turismo de la Gobernación de Boyacá. 
La Provincia de Sugamuxi es un destino que refleja actividad cultural debido a la presencia de raíces ancestrales pertenecientes a pueblos indígenas, como los mitos, arte religioso y republicano, literatura y arquitectura hispanoamericana. Todos los pueblos poseen tradición y son patrimonio cultural departamental y nacional por las raíces indígenas, centros arqueológicos, cultura prehispánica y la historia independentista. Tienen industria hotelera con oferta amplia y variada, disponen de instalaciones deportivas y facilidades para practicar deportes. Existe amplia y diversa oferta comercial. Se organizan y se celebran espectáculos culturales reconocidos a nivel nacional y departamental. Ofrece diversidad de atractivo ecológico. No dispone de amplia oferta de entidades financieras con fácil acceso. Existe amplia cobertura sanitaria. No se puede acceder por vía aérea con facilidad, se puede acceder por carretera de forma fácil, no dispone de una amplia red de carreteras intermunicipales. Los precios de bares, hoteles y restaurantes comparados con los demás destinos a nivel nacional son considerados baratos. La proporción de extranjeros con relación al total de turistas no es elevada. El entorno en general genera seguridad ciudadana y tranquilidad.

Además, se identifican los puntos débiles en donde es indispensable trabajar en la región y las principales fortalezas que se deben tener en cuenta para el análisis estratégico según análisis de expertos.

Como se observa en la Tabla 10, dentro de las debilidades encontradas significativas están la infraestructura, la información, la organización y los lazos del mercado, estas debilidades se tienen que mejorar puesto que el sector turístico se está proyectando como clase mundial.

\section{Tabla 10}

Indicadores de Competitividad Provincia de Sugamuxi

\begin{tabular}{lllc}
\hline \multicolumn{1}{c}{ Actores } & \multicolumn{1}{c}{ Variables } & \multicolumn{1}{c}{ Definición } & $\begin{array}{c}\text { Indicador de los } \\
\text { actores (1 y débil, } 3 \\
\text { medio,4 bueno y 5 } \\
\text { superior }\end{array}$ \\
\hline $\begin{array}{l}\text { Factores de } \\
\text { soporte y } \\
\text { recursos }\end{array}$ & $\begin{array}{l}\text { Infraestructura } \\
\text { en la región }\end{array}$ & $\begin{array}{l}\text { La infraestructura presenta debilidades en cuanto a las } \\
\text { vías terrestres y movilidad, se carece de } \\
\text { infraestructura que pueda prestar un buen servicio al } \\
\text { turista. }\end{array}$ & 1 \\
& $\begin{array}{l}\text { Accesibilidad de } \\
\text { la región }\end{array}$ & $\begin{array}{l}\text { La Provincia de Sugamuxi está ubicada en el centro } \\
\text { del departamento de Boyacá, se caracteriza por } \\
\text { ofrecer un turismo cultural, religioso y de negocios, } \\
\text { por contar con cercanía a las ciudades más }\end{array}$ & \\
& &
\end{tabular}


Factores de Fisiografía atracción

Dirección del destino importantes de Colombia como son: Bogotá D.C, Bucaramanga, los Llanos. La movilidad para el turista es de fácil acceso y de gran importancia para realizar las actividades anteriormente mencionadas.

Desarrollo de las La Provincia de Sugamuxi por su ubicación empresas geográfica, cuenta con varias empresas multinacionales como son: Acerías Paz del Rio, Holcim y Argos, adicional se manejan pequeñas y medianas empresas localizadas en el parque industrial de la ciudad de Sogamoso, por tal razón es Sogamoso la capital de la Provincia y por ende una de las ciudades donde más establecimientos de servicio de alojamiento ofrece al sector turístico.

El departamento de Boyacá cuenta con atractivos naturales considerados patrimonio natural como son el Nevado del Cocuy, la Laguna de Tota, siendo estos dos un centro turístico importante para la afluencia del visitante. En cuanto a la Provincia de Sugamuxi, se cuenta con diversidad de climas y zonas naturales, como son el Páramo de Siscunsi, el Museo arqueológico, entre otros, por tal razón esta zona del país ofrece al turista diversidad y diversos atractivos.

Cultura En cuanto a la cultura se cuenta con municipios religiosos como son Monguí, Topaga y Sogamoso, donde la variedad de templos religiosos son su atracción, culturalmente es considerada la Provincia de Sugamuxi como la más importante para ofrecimiento de historia y belleza natural.

Lazos del En este indicador se presenta una debilidad, puesto mercado que los prestadores del servicio turístico no cuentan con estrategias empresariales que puedan unificar todos estos servicios y así mismo incentivar a los propietarios a mejorar la calidad.

Acontecimientos Esta provincia presenta acontecimientos importantes especialmente en el mes de julio donde se desarrollan el festival del sol y del acero, en la ciudad de Sogamoso el cual ofrece una gran participación de turistas.

Rentabilidad En los últimos años y según estadísticas del informe presentado por la Alcaldía del municipio de Sogamoso, el sector turístico está tomando gran fuerza y así mismo dejando utilidades a los prestadores del servicio, aunque se cuenta con dificultades anteriormente mencionadas.

Marketing Aun se carece de estrategias de publicidad y marketing, es un indicador en el cual se está trabajando fuertemente desde la gobernación del departamento de Boyacá.

Organización Los prestadores de servicio de turismo en la Provincia, funcionan muy bien individualmente, se presentan algunas debilidades para la organización entre varias empresas prestadoras de servicios, lo que genera que el turismo se esté estancando y no se 


\begin{tabular}{|c|c|c|c|}
\hline & & $\begin{array}{l}\text { produzca un valor agregado a los diferentes servicios } \\
\text { que se brindan. }\end{array}$ & \\
\hline \multirow{4}{*}{$\begin{array}{l}\text { Determinantes } \\
\text { restrictivos }\end{array}$} & Información & $\begin{array}{l}\text { Se carece de cualificación por parte del recurso } \\
\text { humano de cada empresa prestadora de servicio, } \\
\text { especialmente en la atención al cliente. }\end{array}$ & 2 \\
\hline & Servicio & $\begin{array}{l}\text { El servicio prestado es bueno, pero falta mejorar } \\
\text { aspectos relevantes para incrementar la calidad. }\end{array}$ & 3 \\
\hline & Localización & $\begin{array}{l}\text { La localización es buena, de acuerdo a la ubicación } \\
\text { geográfica. }\end{array}$ & 5 \\
\hline & Seguridad & $\begin{array}{l}\text { El departamento de Boyacá y la Provincia de } \\
\text { Sugamuxi, cuenta con seguridad, lo cual brinda al } \\
\text { visitante tranquilidad. }\end{array}$ & 5 \\
\hline
\end{tabular}

Fuente: elaboración propia a partir del modelo de Crouch y Ritchie (1999).

Se observa falencias en cuanto a la infraestructura desde el punto de vista vial, ya que a pesar de la construcción de la doble calzada entre la capital Bogotá y el departamento, aún se presentan problemáticas de esta índole al interior de la Provincia, adicional a esto se presentan falencias de organización empresarial enfocada específicamente en la asociatividad, dado que cada sector turístico realiza su labor independiente, generando limitaciones de marketing ya que no se presentan a nivel general planes turísticos que permitan integrar todos los procesos.

\section{Conclusiones}

Con el análisis documental, se indagaron los diferentes estudios enfocados en competitividad en el sector turístico, abordándolo desde el ámbito general a lo particular para mostrar como resultado la importancia de este sector para la economía mundial. Desde el contexto nacional, se evidencia el fortalecimiento del turismo en la participación de la economía, aunque se detallan los esfuerzos por parte del Estado para mejorar las políticas enfocadas en el sector turístico. Con esto, se exponen debilidades que se presentan en algunas regiones y la baja competitividad de las mismas por falta de recursos económicos, conocimiento y estrategias por los actores que intervienen en los procesos. En cuanto a la evaluación de los modelos de competitividad, se adapta el modelo de Crouch y Ritchie (1999) para la población objeto de estudio, ya que se implementan los factores competitivos para la provincia; se puede observar algunas debilidades dentro de las cuales se encuentra el mercadeo turístico, pues esta zona es conocida en temporada de festividades de Semana Santa y fin de año, lo que tiene como consecuencia que sean muy pocas las fechas para posicionar este sector en la economía regional; igualmente, la infraestructura es un tema débil, ya que no es viable para los actores la inversión dada la baja receptibilidad de los turistas. 
Se sugiere para investigaciones futuras proponer estrategias empresariales especialmente en la asociatividad de los diferentes actores que desarrollan el sector turístico en la Provincia; con el fin de posicionar este importante renglón económico a nivel nacional y así mismo mejorar la competitividad turística.

\section{Referencias}

(1) Alberca, P. y Parte, L. (2013). Evaluación de la eficiencia y la productividad en el sector hotelero español: un análisis regional. Investigaciones Europeas de Dirección y Economía de la Empresa, 19(2), 102-111. https://doi.org/10.1016/j.iedee.2012.10.004

(2) Andrades, L., Sánchez, M., \& Pulido, J. (2014). Tourism destination competitiveness from a demand point of view: an empirical analysis for Andalusia. Tourism Analysis, 19(4), 425-440.

(3) Bagarić, L., \& Žitinić, D. (2013). Competitiveness of kvarner region: challenges for destination management and branding. Tourism and Hospitality Management, 19(2), 217-231.

(4) Berki, M., \& Gonda, T. (2006). Positioning of urban scenes of cultural tourism in Hungary. Foldrajzi Ertesito, 55(1-2), 127-140.

(5) Bolaky, B. (2011). La competitividad del turismo en el Caribe. http://repositorio.cepal.org//handle/11362/11459

(6) Cámara de Comercio de Sogamoso. (2017). Diseño de producto turístico para la Provincia de Sugamuxi. https://camarasogamoso.org/diseno-de-producto-turistico-para-la-provincia-desugamuxi/

(7) Castellanos, C., Hernández, Y., Castellanos, J. y Campos, L. (2014). La competitividad del destino turístico Villa Clara, Cuba: Identificación de sus factores determinantes mediante análisis estructural (MIC-MAC). Estudios y Perspectivas en Turismo, 23(2), 250-277.

(8) Chen, W. J. (2013). Factors influencing internal service quality at international tourist hotels. International Journal of Hospitality Management, 35, 152-160. https://doi.org/10.1016/j.ijhm.2013.06.004

(9) Consejo Nacional de Política Económica y Social [CONPES]. (2008). Documento Conpes 3527 - Política Nacional de Competitividad y Productividad. https://colaboracion.dnp.gov.co/CDT/Conpes/Econ\%C3\%B3micos/3527.pdf 
(10) Consejo Privado de Competitividad. (2019). Informe Nacional de Competitividad 2018-2019. https://compite.com.co/wp-content/uploads/2018/10/CPC_INC_2018-2019_Web.pdf

(11) Crouch, G., \& Ritchie, R. (1999). Tourism, competitiveness and societal prosperity. Journal of Business Research, (44), 137-152.

(12) Firgo, M., \& Fritz, O. (2017). Does Having the Right Visitor Mix Do the Job? Applying an Econometric Shift-Share Model to Regional Tourism Developments. The Annals of Regional Science, 58, 469-490. https://doi.org/10.1007/s00168-016-0803-4

(13)Gobernación de Boyacá. (2020). Secretaría de Turismo. https://www.boyaca.gov.co/Secretaríaturismo/

(14) Guzmán, F. A., Cuervo, H. A. e Ibarra, A. (2016). La innovación en el sector turismo como ventaja comparativa entre los países GEM de la Alianza del Pacífico. Revista ESPACIOS, 37(8), 21. http://www.revistaespacios.com/a16v37n08/16370822.html

(15) Ibáñez, R. (2011). Diagnóstico de la calidad y competitividad del sector turístico en México. Cuadernos de Turismo, (28), 121-143. http://uaemex.redalyc.org/articulo.oa?id=39821278007

(16) Kozak, M. \& Andreu, L. (Eds.). (2006). Progress in tourism marketing. Routledge. https://doi.org/10.4324/9780080480480

(17)Lall, S. (2001). Competitiveness Indices and Developing Countries: An Economic Evaluation of the Global Competitiveness Report. World Development, 29(9), 1501-1525.

(18) Ministerio de Comercio, Industria y Turismo [MinCIT]. (2014). Norma Técnica NTS-TS Sectorial Colombiana 002 Establecimientos de Alojamiento y Hospedaje (EAH) Requisitos de sostenibilidad. https://www.mincit.gov.co/CMSPages/GetFile.aspx?guid=f200f357-97f9-408aa2c1-07e1a929c1fc

(19) Narváez, M. y Fernández, G. (2009). Indicadores de competitividad para destinos turísticos en el marco de la sostenibilidad: un análisis aplicado a La Península de Paraguaná. Revista Venezolana de Análisis de Coyuntura, 15(2), 101-119. http://www.redalyc.org/articulo.oa?id=36412216007

(20) Ochoa, E. A. (2015). Determinantes de la competitividad de un destino turístico: caso Villa de Leyva. Visión Empresarial, 1(1), 30-48. https://doi.org/10.24267/24629898.91

(21) Organización Mundial de Turismo [OMT]. (2018). Panorama OMT del turismo internacional. UNWTO. https://doi.org/10.18111/9789284419883

(22) Polanco, J. (2011). Determinantes de un sistema organizacional en red para el desarrollo rural del turismo en Antioquia (Colombia). Cuadernos de Desarrollo Rural, 8(67), 251-274. 
http://www.scielo.org.co/scielo.php?script=sci_arttext\&pid=S0122$14502011000200011 \& \operatorname{lng}=\mathrm{en} \& \operatorname{tlng}=\mathrm{es}$

(23) Porter, M. (2015). Ventaja Competitiva: Creación y sostenimiento de un desempeño superior. Grupo Editorial Patria.

(24) Pulido, J. y Sánchez, M. (2010). Competitividad versus crecimiento en destinos turísticos. Un análisis mediante técnicas multivariantes. Cuadernos de Economía, 33(91),159-181. https://doi.org/10.1016/S0210-0266(10)70061-3

(25) Ritchie, B., \& Crouch, G. (2003). The competitive destination: A sustainable tourism perspective. CABI.

(26) Schwab, K. (Ed.). (2018). The Global Competitiveness Report 2018. World Economic Forum. http://www3.weforum.org/docs/GCR2018/05FullReport/TheGlobalCompetitivenessReport2018 .pdf

(27) Secretaría de Turismo de la Gobernación de Boyacá. (2018). Sistema de Información Turística de Boyacá SITUR. https://situr.boyaca.gov.co/

(28) Urrutia, J. y Cuevas, T. (2016). Redes empresariales en el sector turismo y servicios para la mejora de competitividad en Ciudad Juárez, Chihuahua, México: caso Parque Central Hermanos Escobar y PYMES aledañas. Cuadernos de Turismo, (37), 421-436. https://doi.org/10.6018/turismo.37.256331

(29)Zuñiga, A, y Castillo, M. (2012). Turismo en Colombia: resultados del sector (2007-2010). Magazín Empresarial, 8(15), 67-73.

Cómo citar este artículo: Serrano-Amado, A., Montoya-Restrepo, L. y Amado-Cely, N. (2021). La competitividad turística. Una aproximación desde el Departamento de Boyacá, Colombia. Tendencias, 22(1), 226-253. https://doi.org/10.22267/rtend.212201.162 